\section{Embolisation eines Aneurysmas der Arteria suprarenalis}

Aneurysmen der Viszeralarterien sind nicht unüblich, sie betreffen meistens Leber-, Nieren- oder Milzgefäße und können sich unter dem klinischen Bild eines akuten Abdomens offenbaren (Jorgsen BA, Danish Med Bulletin 1985; 32: 237). In der Literatur werden mehr als 2000 Fälle von Viszeralarterienaneurysmen berichtet, die sich in etwa 115 der Fälle als akutes Abdomen präsentierten (Stanley JC et al., J Vasc Surg 1986; 5: 837).

Über symptomatische Nebennierenarterienaneurysmen wird in der Literatur

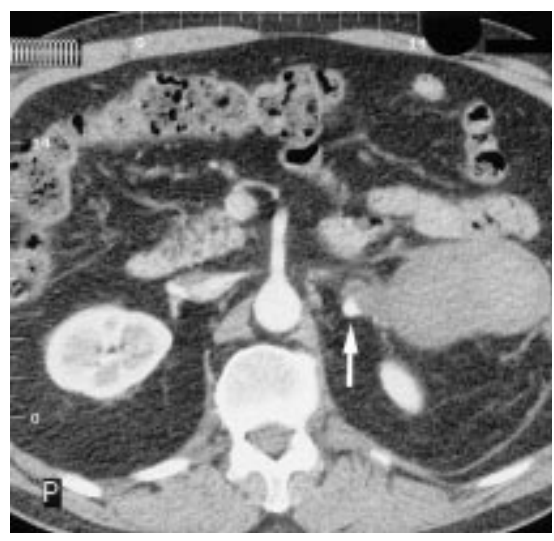

Abb. 1 Kontrastmittelgestützte CT: Retroperitoneales Hämatom in Organisation, Inzidentalom der linken Glandula suprarenalis und kleines Aneurysma der linken A. suprarenalis media (siehe Pfeil).

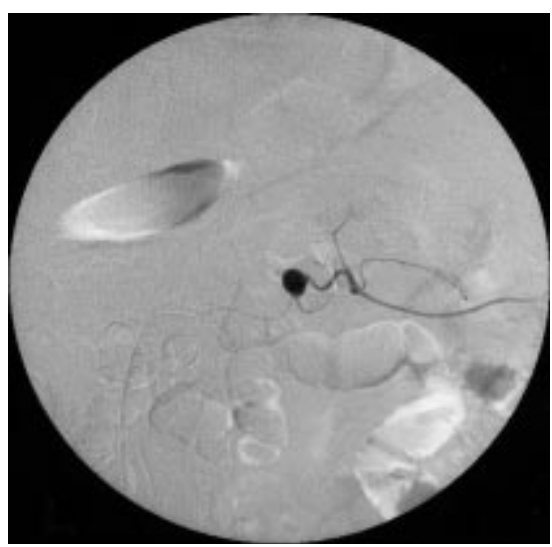

Abb. 2 Selektive DSA der A. suprarenalis media links mit Darstellung des Aneurysmasacks. weltweit lediglich anhand von 4 Patienten berichtet [Birchall D et al., Clin Radiol 1995; 50 (10): 732; Hachulla E et al., J Rheum 1993; 20 (1): 158; Perona F et al., Cardiovasc Interv Radiol 1996; 19: 291; Waterworth $\mathrm{M}$ et al., Lancet 1976; 21; 1 (7956): 424]: Zwei Patienten wurden einer operativen Therapie zugeführt und zwei Patienten einer superselektiven Embolisationstherapie mit Coils.

Im folgenden berichten wir über einen Patienten mit einer symptomatischen Blutung aus einem Aneurysma der linken A. suprarenalis mit Ausbildung eines

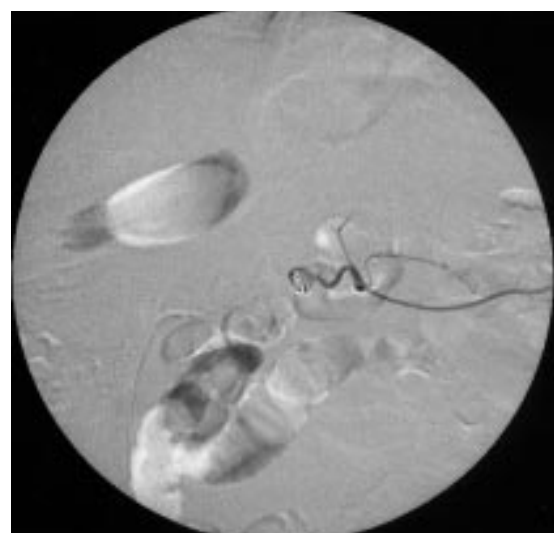

Abb. 3 Positionierung der Mikrocoils rnittels eines Tracker-Katheters in den Nebennierenarterienaneurysmasack.

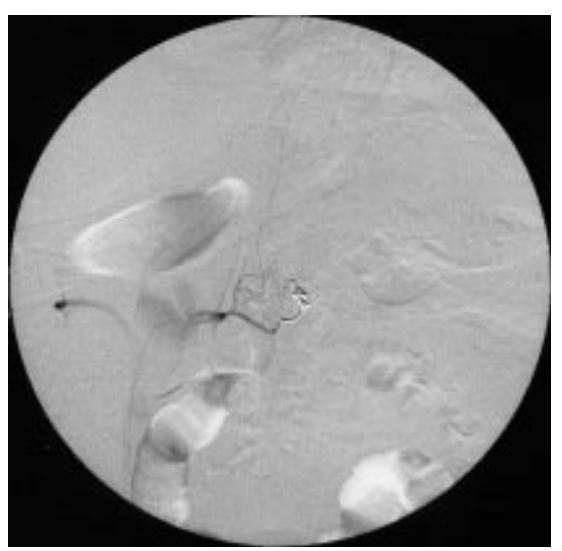

Abb. 4 Angiographische Kontrolle nach Embolisation: Komplettes Thrombosieren des Aneurysmasacks. retroperitonealen Hämatoms und der superselektiven Aneurysmaembolisation mit Mikrocoils.

\section{Fallbeschreibung}

Es handelt sich um einen 61jährigen Patienten, der sich mit erstmaligen starken Dauerschmerzen in der linken Flankenregion in der Notfallambulanz vorstellte. Sonographisch konnte man bei Aufnahme ca. $500 \mathrm{ml}$ freie Flüssigkeit in der Nieren-Milzloge erkennen. Ein Hinweis für eine Nephrolithiasis bzw. Ureterolithiasis fand sich nicht.

Der Patient wurde zur weiteren Abklärung stationär aufgenommen.

Kontrollsonographien zeigten eine zunehmende Organisation und Größenregression der im initialen Sonogramm vorbeschriebenen Flüssigkeitsansammlung in der Nieren-Milzloge. Die Arbeitsdiagnose lautete somit initiale Blutung bzw. Hämatom in Organisation. Zusätzlich zeigte sich sonographisch eine unklare Raumforderung von ca. $2 \mathrm{~cm}$ Durchmesser in der linken Nebennierenregion. Zur weiteren Klärung wurde eine KM-gestützte Computertomographie des Oberbauches veranlaßt.

Auf den Schichtaufnahmen (Abb.1) ließ sich das vermutete ca. $8 \times 7 \times 7 \mathrm{~cm}$ große, teilorganisierte Hämatom in der NierenMilzloge nachweisen. Außerdem fiel in der linken Nebennierenregion eine rundliche Weichgewebsvermehrung von ca. $2 \mathrm{~cm}$ Größendurchmesser und caudomedial dieser Struktur ein rundliches KM-Depot von ca. $0,5 \mathrm{~cm}$ Querschnitt auf. Nach weiterführenden laborchemischen Untersuchungen konnte die RF in der Nebennierenregion einem Inzidentalom zugeschrieben werden, ein maligner Prozeß wurde ausgeschlossen. Zur Klärung der punktförmigen KM-Anreicherung zwischen Aorta und Nebennierenregion im CT wurde eine Angiographie (Abb.2) durchgeführt, die die Verdachtsdiagnose eines Nebennierenarterienaneurysmas bestätigte. Im Angiogramm (Abb. 2) konnte ca. $3 \mathrm{~cm}$ nach dem Abgang der linken A. suprarenalis media ein kleines sacciformes Aneurysma von knapp $0,5 \mathrm{~cm}$ Durchmesser nachgewiesen werden. Das Trägergefäß wies eine Lumenweite von ca. $1 \mathrm{~mm}$ auf.

Das ausgedehnte Hämatom in der linken Nebennierenregion konnte somit auf eine Blutung aus dem kleinen Aneurys- 
ma der linken A. suprarenalis media zurückgeführt werden.

Aufgrund des Blutungsrisikos und auf Wunsch des Patienten erfolgte nach Abwägen aller Risiken der Entschluß zu einer Embolisation des Aneurysmas. Transfemoral wurden in coaxialer Technik mittels eines Tracker-Katheters 3 Mikrocoils (.018 Inch-Durchmesser gestreckt, $7 \mathrm{~mm}$ Spiraldurchmesser aufgerollt und 2,1 cm Länge) in den Aneurysmasack plaziert (Abb.3). Bereits nach wenigen Minuten konnte in den Kontrollangiographien eine Thrombosierung mit komplettem Verschluß des Aneurysmasacks und des dahinter gelegenen Gefäßabschnittes dokumentiert werden (Abb. 4).

\section{Diskussion}

In der Literatur wird von 4 Fällen einer Blutung aus einem Aneurysma der A. suprarenalis berichtet [Birchall D et al., Clin Radiol 1995; 50 (10): 732; Hachulla E et al., J Rheum 1993; 20 (1): 158; Perona $F$ et al., Cardiovasc Interv Radiol 1996; 19: 291; Waterworth $M$ et al., Lancet 1976; 21; 1 (7956): 424]. Bei allen Patienten wurde eine ähnliche Symptomatik mit plötzlich einsetzenden Dauerschmerzen in der Flankenregion beschrieben. Sonographie und CT wiesen jeweils das retroperitoneale Hämatom auf, die Angiographie sicherte die Blutungsquelle.

Im ersten berichteten Fall [Waterworth M et al., Lancet 1976; 21; 1 (7956): 424] wurde aufgrund des Verdachts einer Ulkusblutung notfallmäßig laparotomiert. Intraoperativ konnte als Ursache für die Blutung ein rupturiertes sackförmiges Nebennierenarterienaneurysma von $12 \mathrm{~mm}$ Länge und $10 \mathrm{~mm}$ Breite identifiziert werden. Histologisch zeigte sich lediglich eine verschmälerte Lamina elastica interna, ansonsten keine weiteren Auffälligkeiten.

Im zweiten Fall [Hachulla E et al., J Rheum 1993; 20(1): 158] wird ein Patient mit Polyarteriitis nodosa beschrieben, bei dem die Blutung aus einem ca. $0,5 \mathrm{~cm}$ breiten Aneurysma der A. suprarenalis inferior durch selektive Embolisation mit Mikrocoils gestoppt wurde.

Der dritte berichtete Fall [Birchall D et al., Clin Radiol 1995;50(10): 732] beschreibt die operative Versorgung eines $4 \mathrm{~cm}$ breiten Aneurysmasacks der A. suprarenalis inferior. Histologisch konnten am Präparat atheromatöse Veränderungen festgestellt werden.

Im letzten Fallbericht (Perona F et al., Cardiovasc Interv Radiol 1996; 19: 291) wird die Embolisation eines ca. $1 \mathrm{~cm}$ breiten Aneurysmas der A. suprarenalis inferior mit Polyvinyl-Alkohol-Partikeln (Durchmesser 250-335 $\mu \mathrm{m}$ ) beschrieben.

Als Ursache eines akuten Abdomens stellen rupturierte Viszeralarterienaneurysmen eine sehr kleine Entität dar, sollten aber in weitergehende differentialdiagnostische Erwägungen miteinbezogen werden. CT und Angiographie haben hierbei die diagnostische Schlüs- selfunktion. Therapeutisch sollte die Embolisation als interventionell-radiologisches Verfahren in Betracht gezogen werden.

1. Rupturen von Nebennierenarterienaneurysmen können sich unter dem klinischen Bild eines akuten Abdomens präsentieren.

2. CT und Angiographie besitzen bei V.a. Nebennierenarterienaneurysmen eine diagnostische Schlüsselfunktion

3. Die Embolisation von rupturgefährdeten Nebennierenarterienaneurysmen sollte als interventionellradiologisches Verfahren in Betracht gezogen werden.

K. Brenner-Maucher, J. Rieger, E. Mangel, G. Leinsinger, K. -J. Pfeifer, München 